\title{
Artefact
}

Techniques, histoire et sciences humaines

$11 \mid 2019$

Pannes et accidents (XIX $\mathrm{X}^{\mathrm{e}} \mathrm{XXI^{ \textrm {e } }}$ siècle)

\section{Lise Saussus, avec la collaboration de Nicolas Thomas, Marisa Pirson et Benjamin Jagou, Travailler le cuivre à Douai au XIII ${ }^{e}$ siècle, histoire et archéologie d'un atelier de proximité}

Douai, Association Les amis d'Arkeos, Louvain-la-Neuve, Université catholique, 2019

\section{Michel Pernot}

\section{(2) OpenEdition}

\section{Journals}

Édition électronique

URL : http://journals.openedition.org/artefact/5077

DOI : $10.4000 /$ artefact.5077

ISSN : 2606-9245

Éditeur :

Association Artefact. Techniques histoire et sciences humaines, Presses universitaires du Midi

Édition imprimée

Date de publication : 15 décembre 2019

Pagination : 341-343

ISBN : 978-2-8107-0667-9

ISSN : 2273-0753

Référence électronique

Michel Pernot, «Lise Saussus, avec la collaboration de Nicolas Thomas, Marisa Pirson et Benjamin Jagou, Travailler le cuivre à Douai au xIII siècle, histoire et archéologie d'un atelier de proximité ", Artefact [En ligne], 11 | 2019, mis en ligne le 27 novembre 2020, consulté le 30 novembre 2020. URL : http:// journals.openedition.org/artefact/5077 ; DOI : https://doi.org/10.4000/artefact.5077

Ce document a été généré automatiquement le 30 novembre 2020.

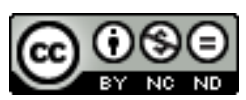

Artefact, Techniques, histoire et sciences humaines est mise à disposition selon les termes de la Licence Creative Commons Attribution - Pas d'Utilisation Commerciale - Pas de Modification 4.0 International. 


\section{Lise Saussus, avec la collaboration de Nicolas Thomas, Marisa Pirson et Benjamin Jagou, Travailler le cuivre à Douai au XIII ${ }^{e}$ siècle, histoire et archéologie d'un atelier de proximité}

Douai, Association Les amis d'Arkeos, Louvain-la-Neuve, Université catholique, 2019

Michel Pernot

\section{RÉFÉRENCE}

Lise Saussus, avec la collaboration de Nicolas Thomas, Marisa Pirson et Benjamin Jagou, Travailler le cuivre à Douai au XIII siècle, histoire et archéologie d'un atelier de proximité, Douai, Association Les amis d'Arkeos, Louvain-la-Neuve, Université catholique, 2019, $277 \mathrm{p}$.

1 Publié très récemment, ce livre est consacré à l'exploitation de données inédites issues de la fouille des vestiges d'un atelier médiéval de travail du cuivre, situé rue SaintJulien à Douai, dans le département du Nord. Collectés lors d'opérations réalisées par le Service archéologique de la ville entre 1983 et 1987, ces documents n'avaient pas été publiés faute de spécialiste de la métallurgie des métaux et alliages non-ferreux pour la période considérée. Le très soigneux travail, construit et principalement réalisé par Lise saussus, aboutit à une publication de haut niveau.

2 Pour cause de recyclage des matériaux, les témoins qui nous parviennent des productions en métal des sociétés du passé sont infimes. Méconnaissant cette réalité, les historiens de l'art considéraient généralement Douai - « ville sans lutrin, sans fonts baptismaux, sans chandelier dignes de l'attention »- comme un lieu sans travail des 
alliages cuivreux. Les récentes découvertes archéologiques démontrent qu'il faut corriger cette vision réductrice qui considère seulement les productions des grands centres, principalement situés dans la vallée de la Meuse, tels Dinant et Bouvignes; l'importance de l'activité de ces hauts lieux est incontestable mais des ateliers sont clairement présents en bien d'autres endroits.

Mêlant l'exploitation de la source textuelle avec celle des vestiges matériels du travail des hommes de métier, Lise saussus reconstruit une image complexe d'un pan de la société médiévale qui a fonctionné à Douai au XIII ${ }^{\mathrm{e}}$ siècle. Dans un examen approfondi de l'histoire du quartier et du cadre de la découverte, les vestiges immobiliers, essentiellement des restes de murs et de fours, sont analysés pour retracer l'évolution des bâtiments et de l'organisation des activités artisanales. Les mobiliers liés au travail métallurgique, c'est-à-dire les fragments de céramique de moules et de creusets ainsi que les chutes de métal, sont présentés dans un premier temps. Leur étude est ensuite reprise systématiquement dans un chapitre sur les techniques mises en œuvre dans l'atelier ; la réalisation de moules pour la fonderie et le travail du métal par battage et recuit sont bien expliqués en associant des textes et des images du monde médiéval avec des schémas explicatifs bien construits. La dualité - intemporelle car liée aux matériaux métalliques - des possibilités de mise en forme du métal, soit par moulage soit par déformation, est fort bien illustrée par la coexistence de marmites massives, réalisées par fonderie, et de chaudrons en tôle obtenus par des passes de martelage alternées de recuits.

Le chapitre consacré aux techniques de réparation d'objets métalliques est également très didactique ; il associe l'étude des témoins archéologiques, tels des rivets en tôle, à des photographies d'objets médiévaux réparés. Encore une fois la présentation de citations de documents écrits et d'images sensiblement contemporaines de l'activité de l'atelier contribue au réalisme des restitutions proposées.

5 Écrit en collaboration avec Nicolas Thomas (auteur du remarquable travail pionnier sur le site de l'hôtel de Mongelas à Paris), le chapitre qui traite des analyses de la composition des alliages met bien en relief les stratégies qui guident les hommes dans leurs choix; en effet, ils doivent nécessairement accorder le type d'alliage mis en œuvre avec le procédé dominant de mise en forme. La complexité des problèmes, résolus avec succès par les artisans - qui, rappelons-le, disposent de tests mais non de moyens d'analyses - pour tenter de contrôler les teneurs en éléments d'alliage ajoutés au cuivre, l'étain, le zinc et le plomb, est discutée avec pertinence.

6 Un chapitre, écrit par Benjamin Jagou, étudie les vestiges du travail du fer, matériau indispensable au travail du cuivre à la période médiévale; il faut nécessairement assurer la confection et l'entretien d'outils en fer, voire en acier. Les témoins du forgeage - phase de travail à chaud du fer - indiquent l'usage de charbon de terre ; l'emploi de ce dernier, en parallèle à celui du charbon de bois pour le travail du cuivre, continuera d'alimenter une fort intéressante discussion dans un champ de recherche encore largement ouvert. L'étude des vases en céramique, sans lien avec le travail du métal, réalisée par Marisa Pirson, constitue un autre court chapitre. Les formes et les décors de ces récipients d'usage domestique confortent la datation au XIII ${ }^{\mathrm{e}}$ siècle du fonctionnement de l'atelier.

7 Avant de conclure, Lise saussus examine la répartition spatiale des vestiges. Les modalités de l'organisation de l'espace artisanal, ainsi que de son évolution, sont ainsi, 
dans la mesure du possible en raison du caractère lacunaire normal des données, mises en évidence.

Parmi un large ensemble d'éloges, une critique doit cependant être formulée. Il est dommage que les fragments dits de "plomb» (Tableau 1, p. 84) n'aient pas été l'objet de plus de considération, en particulier car il n'est pas certain qu'il s'agisse de ce matériau. En effet, l'étain n'est pas seulement un élément d'alliage avec le cuivre ; il est aussi employé non allié, ou en alliage avec le plomb, pour réaliser des assemblages par brasure et des revêtements de surface par étamage. Des analyses permettraient de préciser les choix faits par les métallurgistes (plomb, étain, alliages étain-plomb) ; cela ouvrirait d'intéressantes discussions sur les pratiques autres que le rivetage et sur la protection des surfaces internes des récipients culinaires en alliages à base de cuivre en relation avec la toxicité de ce dernier.

Les conclusions de ce travail scientifique extrêmement méticuleux sont parfaitement claires en démontrant l'existence, et le fonctionnement, d'un atelier qualifié « d'atelier de proximité ». Les lieux de travail de ce type - certainement beaucoup plus répandus qu'on le suppose encore aujourd'hui - jouent, en parallèle aux grands centres de production, un rôle absolument non négligeable dans l'économie médiévale du cuivre. L'étude très approfondie des techniques de réparation, des défauts de fabrication ou des percements dus à l'usage, explore la réalité de l'entretien souvent négligée devant le prestige de la production initiale. Il est évident que cet ouvrage restera longtemps une référence, et pas uniquement dans l'univers médiéval. D'une part, il associe l'exploitation, quasi exhaustive, des diverses sources documentaires - vestiges matériels, écrits et images - ; d'autre part, il met en évidence l'existence d'ateliers, probablement sans liens avec des productions prestigieuses, mais d'une indispensable utilité dans la vie quotidienne.

\section{AUTEURS}

\section{MICHEL PERNOT}

Université Bordeaux-Montaigne, IRAMAT (UMR 5060) 